\title{
Annotating and quantifying narrative time disruptions in modernist and hypertext fiction
}

\author{
Edward Kearns \\ National University of Ireland, Galway \\ edward. kearnsenuigalway. ie
}

\begin{abstract}
This paper outlines work in progress on a new method of annotating and quantitatively discussing narrative techniques related to time in fiction. Specifically those techniques are analepsis, prolepsis, narrative level changes, and stream-of-consciousness and free-indirectdiscourse narration. By counting the frequency and extent of the usage of these techniques, the narrative characteristics of different works from different time periods and genres can be compared. This project uses modernist fiction and hypertext fiction as its case studies.
\end{abstract}

\section{Introduction}

This project annotates and analyses a specific combination of narrative techniques that have not been treated in this way before. Its understanding of narrative emanates from English literary studies, but is applied using a methodology that combines the methods of that field with digital humanities methods, specifically annotation. There has been work on annotating narrative features, but not featuring the combination of narrative levels, time disruptions, and subjective narration. This paper shows a new way for this to be done using a custom XML schema and analysis of preliminary data gleaned from the application of that schema. This process can be used to compare fiction from any genre or time period because most of the techniques involved in the annotation scheme have been used in fiction for centuries. This project applies the process to modernist fiction (experimental novels from the early 20th century) and hypertext fiction (texts from the last four decades which are designed specifically to be read on a computer) because comparing them quantitatively can help to trace the relationship between the two genres which has been proposed in literary theory. This is work in progress, and this paper's analysis will mainly focus on the use of analepsis, which is one of the techniques of disrupting narrative time.

\section{Description of project and research question}

This project seeks to determine whether a valid and useful system of encoding narrative characteristics related to time in fiction can be developed. It then asks how the narratives thus encoded can be quantitatively analysed, using modernist and hypertext fiction as case studies. The narrative techniques which this project addresses are analepsis, prolepsis (flashbacks and flashes forward in story time, respectively, and collectively referred to as anachronies), changes in narrative level (stories within stories), and two kinds of subjective narration: stream of consciousness (prose that reports a character's thoughts directly and continuously, often ignoring rules of grammar in order to do so) and free indirect discourse (prose that still uses a separate narrator and third-person sentences, but in which the words are affected by the biases and perspective of a particular character). If one is comparing transitions in narrative time, then one must determine to what extent those things are happening, and how frequently. The workflow consists of annotating the novels and hypertexts; counting the encoded instances of anachrony, subjective narration, and changes in narrative level; determining where these phenomena appear in each text; comparing those patterns between the texts; and then going back to the texts themselves to read what is going on at particular points of activity, to see why these changes in the narration are occurring. Works are regularly compared in literary studies, and narrative theory is often used to compare the portrayal of time in fiction works of different kinds. What this project adds to that is a more measurable 
way of describing the structural positioning and role of of narrative time disruptions in fiction.

\section{Related quantitative work}

Several annotation schemes and digital humanities literary studies have influenced this project. Preexisting annotation schemes have shown how some similar narrative aspects can be encoded. NarrativeML is intended for encoding the goals of characters and narrators, aspects of pacing in narrative, and embedded narratives (Mani, 2013). Its tagset has a small number of elements, but a large amount of attributes and values that can be added to those elements. This makes NarrativeML good for its intended use, but its complexity is not necessary for the goals of this project, and it does not address the combination of narrative time, subjective narration, and changes in level. ProppML is useful as another example of a system for manually encoding some narrative features in fiction (Yarlott and Finlayson, 2016), but it is intended for folk tales with formulaic plots and it is also quite complex with many attributes for each element. Narrative time is the primary focus of TimeML (Saurí et al., 2006). Its Timex 3 and Tlink tags allow numbers to be assigned in their values for recording the relative order of events, but there are no specific tags for analepsis or prolepsis, and the annotation scheme is not concerned with subjective narration or narrative levels so there are no tags for them either. These kinds of schemes have shown that narrative characteristics can be encoded, but have not addressed the specific ones that this project addresses.

The Narrelations tool represents annotated narratives as a diagram consisting of concentric rings (Schwan et al., 2019). It is excellent for showing how many narrative levels a story has, and the proportion of story length spent in each level. However, it does not show the degree to which narrative level changes and anachronies are happening - how many words are being encompassed by these techniques. It cannot show relative peaks or troughs in the usage of those techniques over the course of a text, only binary information on whether they are happening or not. It does not feature stream-ofconsciousness or free indirect discourse, or display word counts. As such, it too does not address the specific pieces of information that this project requires.

Some digital humanities projects have shown how quantitative methods can be useful for com- paring fiction works individually and in corpora. Ramsay (2011) compared sections of The Waves by Virginia Woolf against each other, noting the difference in the vocabulary used in sections narrated by the different characters, and what that can tell us about those characters. Jockers (2014) has shown how to visually represent the relative similarities and differences in prose style of a corpus of nineteenth- and twentieth-century novels using cluster dendrograms. Clement (2012) uses visualisations of the frequency of usage of the word "one" increasing dramatically in the second half of Gertrude Stein's The Making of Americans, just as the usage of the word "I" becomes far less frequent. Using this graph Clement shows definite and deliberate structural change in the second half of the novel, driven by its syntax. This illustrates how quantitative measures can help to interrogate the structure of fiction texts, as well as the style of their prose. Text-mining does not work for this project because the narrative concepts being addressed require too much critical interpretation for a computer program to detect, but these practices show the insights that can be gained from comparing data gleaned from fiction works, and then applying those insights to further literary study of those novels.

\section{Data}

The corpus of this project contains six modernist novels and seven pieces of hypertext fiction. The modernist novels are To the Lighthouse by Virginia Woolf, Tender is the Night by F. Scott Fitzgerald, Pointed Roofs by Dorothy Richardson, The Sound and the Fury by William Faulkner, Three Lives by Gertrude Stein, and The House in Paris by Elizabeth Bowen. They have been selected because their publication dates span from 1909 to 1935, and because they feature a wide range of prose and narrative styles. The hypertext fictions chosen are Uncle Roger by Judy Malloy, Twelve Blue by Michael Joyce, my body - a Wunderkammer by Shelley Jackson, Seed by Joanna Walsh, Voyage Into the Unknown by Roderick Coover, Victory Garden by Stuart Moulthrop, The Jew's Daughter by Judd Morrissey and Lori Talley. Similarly, these texts span from 1986 to 2017 and have a variety of different writing styles and narrative structures. Modernist and hypertext fiction often share characteristics such as narrative fragmentation, the effort to make a "significant formal break with the tradi- 
tions that preceded" them (Rettberg, 2018), while still seeking "inspiration and validation in a literary past" (Pressman, 2014). They also share a certain "narrative complexity, and an aesthetic of difficulty" (Pressman, 2014).

\section{Methodology}

In order to compare narrative features in a structured way across different texts, it is necessary to count how much and how frequently those features were occurring. Annotating each occurrence of those features was the way to do this, and this project's combination of tags is necessary so that they can be interwoven in a way that mimics how the narrative techniques are interwoven in the fiction. XML has been used for the annotation.

The elements in the annotation scheme are: Analepsis, Prolepsis, Level (with the attribute 'degree', to which integers can be assigned for each narrative level), SOC (for stream-ofconsciousness), and FID (for free indirect discourse). Each tag can be used on its own, or nested inside one another. Due to the modular structure of this system, the annotation routine does not have to follow a particular order, and not every tag needs to be used. The tags can be applied as necessary. The criteria for beginning and ending annotation spans is the same for all tags. The annotation should begin at the point in the text where the narrative transitions either to a higher or lower level, a different point in time, or into or out of stream-ofconsciousness or free-indirect-discourse narration. This can occur in the middle of a sentence, and can continue into another sentence, paragraph, or chapter. The annotation is ended when the phenomenon ends and the narration changes to a different level, transitions to a different point in time, or changes back to omniscient narration after its spell in more subjective narration has ceased.

Once the texts have been encoded, a number of features are measured quantitatively. They are: (1) numbers of each of the analepsis, prolepsis, narrative level, stream-of-consciousness and free indirect discourse tags in each text, both as total numbers and as percentages relative to the total word counts of each text; (2) how frequently those tags are occurring relative to word counts; (3) numbers of words contained within all tags relative to the total word counts of whole texts, pages, lexias, chapters, and sections; (4) which tags are occurring inside other tags. The analyses in this paper use the first two of those measures.

The scope and budget of the project does not allow for multiple annotators to be hired; as such all text encoding was completed by one annotator, the author. However, the project's schema has been tested for inter-annotator agreement using a separate, smaller corpus, and compared against other similar annotation guidelines that focus on narrative levels, as part of the Systematic Analysis of Narrative Texts through Annotation (SANTA) project. Gius et al. (2019) explain that SANTA "employed the metric $\gamma$ (gamma)" so that they could "compare evaluation schemes with different complexities and to avoid favouring more simple schemes (if the scheme is simpler, chance agreement is higher)". The IAA result for my guidelines was 0.24 in gamma (Willand et al., 2019). By normal IAA measures, 0.24 is quite low (Mathet et al., 2015), but all of the scores in the SANTA project were similarly low. The IAA for my guidelines was the third-highest of the eight annotation guidelines, with results ranging from 0.05 to 0.30 . Further rounds of evaluation are pending in that project.

Recording and analysing number and frequency of narrative features related to time allows direct comparisons of the narrative strategies and structures of literary texts from different genres and time periods. We can see how different or similar these texts are in their treatment of narrative time, in a way that is different to what can be achieved with traditional literary analysis alone. However, that literary analysis is more important, and these quantitative measures do not replace that; rather they are used in concert with it, as can be seen in the next section of this paper. The data only ever serves to lead us back to reading the texts.

\section{Preliminary results and discussion}

These examples are small parts of the overall project, and work is ongoing to develop further comparisons, but for now we can look at all of the texts in the corpus using a basic measure, and then do some more detailed analysis of a select few texts which have characteristics in common. One of the first things to consider across the corpus - and the quantitative measure numbered (1) in the methodology - is how frequently each tag is occurring in each text, relative to the word counts of those texts. To achieve a relative comparison across texts of different lengths, normalised frequency has been used, which is simply the number of a times a particular 
tag occurs in a text, divided by the total word count of that text, with that result multiplied by 10,000. This calculation comes from McEnery and Hardie (2011), and the base of normalisation of 10,000 is essentially arbitrary, but is somewhat similar to the length of the texts in this corpus, most of which are in the tens of thousands of words. Figure 1 in this paper shows the normalised frequency for the analepsis tag across all texts in the corpus. Analepsis is used in all of these examples because it is the most significant tag for the themes of the project, but these measures can be made with all of the tags in the schema.

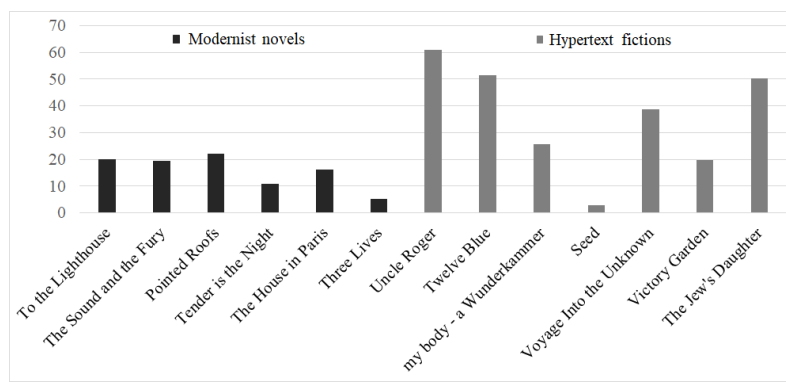

Figure 1: Normalised frequency of analepsis tags, for all texts ((Total no. of analepsis tags / Total no. of words in a text) $x 10,000$ )

As we can see, the modernist novels tend to have comparable frequency of analepsis tags occurring; four of the six novels have normalised frequencies close to 20. The other two, Tender is the Night and Three Lives, both have long sections that are wholly encompassed by one analepsis tag, so their less frequent occurrence of tags does not reflect the large proportion of their narratives that occurs inside analepsis.

For a clearer analysis of these narratives, we need to examine the numbers of words contained within analepsis tags and their placement in the text. Tender is the Night and The House in Paris are useful as an example for this comparison because both novels are divided into three long sections, beginning in the present, then flashing back about ten years in their middle sections, then returning to the present in their final portion. Those long middle analepses in turn contain analepses of their own.

In both of these novels, there are fewer words in analepsis tags at the beginning and end, while the large amount of words within analepsis in their middle sections is clearly evident. The difference is with the analepsis within analepsis. In The House in Paris, the long flashback in the middle has sev-

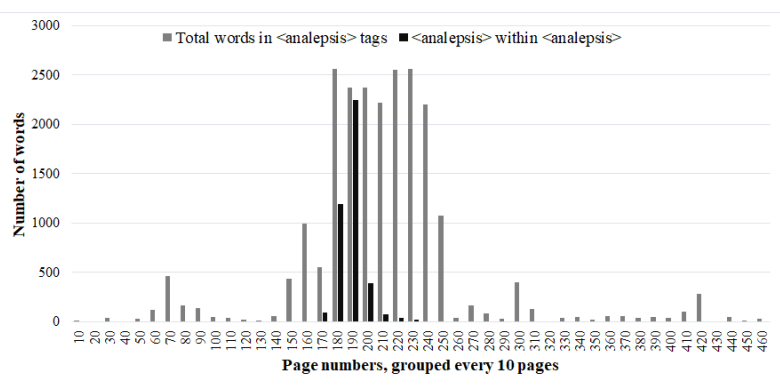

Figure 2: Words in analepsis in Tender is the Night

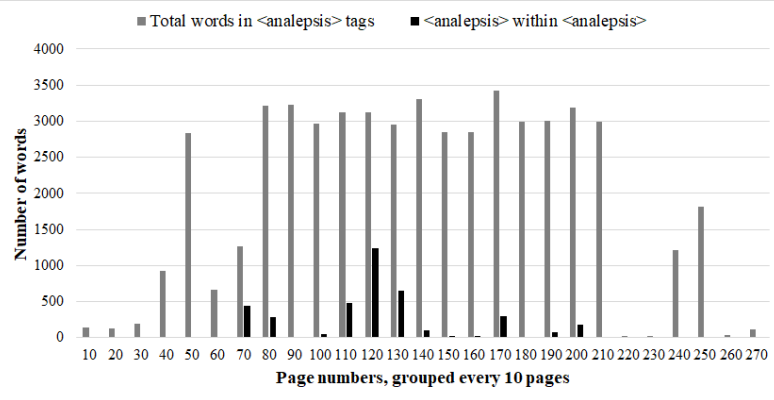

Figure 3: Words in analepsis in The House in Paris

eral small clusters at various points throughout it, rather than the large cluster of analepsis-withinanalepsis that we see at the beginning of the long flashback in Tender is the Night. The dispersed analepses in Bowen's novel mimic the natural references to the past that occur in memory (or storytelling), while Fitzgerald's novel shows a more deliberate construction of particular flashbacks, like a Freudian psychoanalyst imposing a narrative on a patient, a subject with which the novel is concerned in its content.

A linear comparison works for linear texts, but hypertexts are usually nonlinear. They can be analysed on the level of individual lexias, but for overall comparisons between hypertexts and modernist novels, one can compare how frequently tags are occurring, and how the two texts compare in terms of words within analepsis tags as percentages of their overall length. To the Lighthouse and Twelve Blue are useful texts to compare because they both use free-indirect discourse narration from the perspectives of many characters, mostly families and groups of friends. Along with those changing narrative perspectives, both texts also often transition backwards and forwards through time and memory. So, their similar content invites one to ask whether they have similarities in narrative form, despite their different genres. As such, Figures 4 and 5 compare the usage of analepsis in these two 
texts.

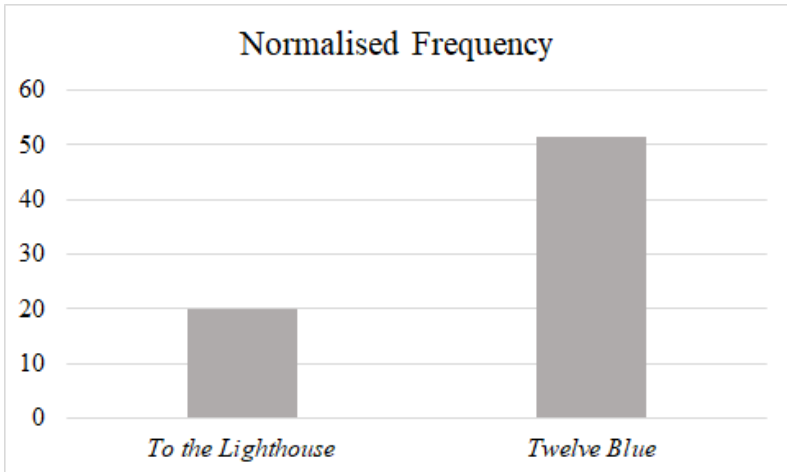

Figure 4: Analepsis tags occur 2.5 times more frequently in Twelve Blue than in To the Lighthouse.

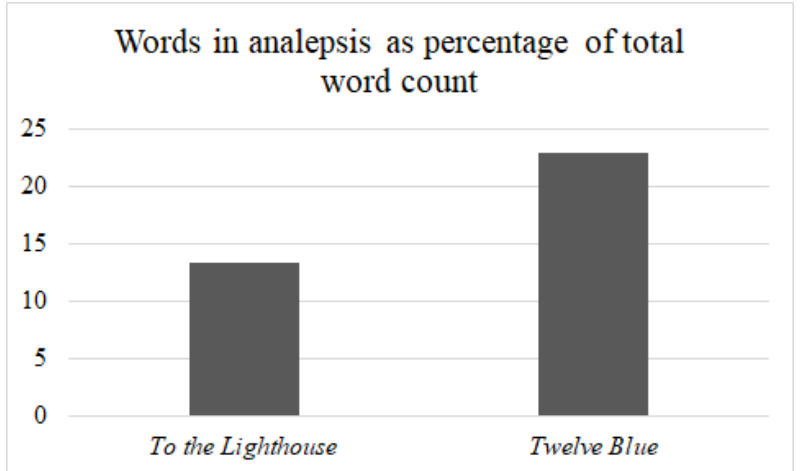

Figure 5: Relative to their total word counts, Twelve Blue only has 1.7 times as many words in analepsis tags than To the Lighthouse

At first it would appear that analepsis is happening overwhelmingly more in Twelve Blue than in To the Lighthouse, but one can see in Figure 5 that the difference is not as great as it initially seems, when word counts are taken into consideration. Still, proportionally, more of Twelve Blue occurs in analepsis than To the Lighthouse. Both texts are concerned with memory and the intersecting lives of groups of people over spans of years. That thematic similarity stands in contrast with their structural differences. The first part of To the Lighthouse is its longest section, setting up most of the significant relationships and story themes; it is the part that the characters spend the shorter final part of the book remembering. Twelve Blue does not work like this; its interweaving-thread structure diverts the reader to different times and places with each click rather than following one story from start to finish. It is more fragmented, with analepses occurring throughout rather than at the end. This invites the question of whether hypertext narratives generally are even more temporally fragmented than those found in modernism, whether hypertext has taken the aesthetics of modernism and experimented with them even further. That is one of the questions that the rest of this project will address.

\section{Conclusion}

Annotation and quantitative analysis allows temporal narrative features to be assessed in a different way than with traditional literary methods. The quantitative perspective enables aspects of literary structure to be measured as well as compared. This methodology can show exactly where spans of narrative disruptions begin and end, and what their length and frequency can tell us about the narrative strategies of a text. In turn this helps us to unpack the "incessant shower of innumerable atoms" with their "sudden lightning flashes of significance" that are the temporally fragmented narratives of modernist and hypertext fiction (Woolf, 1984).

\section{References}

Tanya Clement. 2012. The Story of one: Narrative and Composition in Gertrude Stein's The Making of Americans. Texas Studies in Literature and Language, 54(3):426-448.

Evelyn Gius, Nils Reiter, and Marcus Wielland. 2019. A Shared Task for the Digital Humanities Chapter 2: Evaluating Annotation Guidelines. Journal of Cultural Analytics.

Matthew Jockers. 2014. Text Analysis with $R$ for Students of Literature. Springer, New York. GoogleBooks-ID: K4_IAwAAQBAJ.

Inderjeet Mani. 2013. Computational Modeling of Narrative. Morgan \& Claypool.

Yann Mathet, Antoine Widlcher, and Jean-Philippe Mtivier. 2015. The Unified and Holistic Method Gamma $(\gamma)$ for Inter-Annotator Agreement Measure and Alignment. Computational Linguistics, 41(3):437-479.

Tony McEnery and Andrew Hardie. 2011. Corpus Linguistics: Method, Theory and Practice. Cambridge University Press. Google-Books-ID: 3j3Wn_ZT1qwC.

Jessica Pressman. 2014. Digital Modernism: Making It New in New Media. Oxford University Press, Oxford.

Stephen Ramsay. 2011. Reading Machines: Toward and Algorithmic Criticism. University of Illinois Press. Google-Books-ID: JxPgmAEACAAJ. 
Scott Rettberg. 2018. Electronic Literature. Polity Press, Cambridge, UK ; Medford, MA, USA.

Roser Saurí, Jessica Littman, Bob Knippen, Robert Gaizauskas, Andrea Setzer, and James Pustejovsky. 2006. TimeML Annotation Guidelines Version 1.2.1.

Hannah Schwan, Janina Jacke, Rabea Kleymann, JanErik Stange, and Marian Drk. 2019. Narrelations Visualizing Narrative Levels and their Correlations with Temporal Phenomena. Digital Humanities Quarterly, 013(3).

Marcus Willand, Evelyn Gius, and Nils Reiter. 2019. A Shared Task for the Digital Humanities Chapter 3: Description of Submitted Guidelines and Final Evaluation Results. Journal of Cultural Analytics.

Virginia Woolf. 1984. Modern Fiction. In Andrew McNeille, editor, The Essays of Virginia Woolf. Volume 4: 1925 to 1928. The Hogarth Press, London.

W. Victor H. Yarlott and Mark A. Finlayson. 2016 ProppML: A Complete Annotation Scheme for Proppian Morphologies. In 7th Workshop on Computational Models of Narrative (CMN 2016), volume 53 of OpenAccess Series in Informatics (OASIcs), pages 8:1-8:19, Dagstuhl, Germany. Schloss DagstuhlLeibniz-Zentrum fuer Informatik. 